\title{
Generating Cosmological Solutions from Known Solutions*
}

\author{
Hernando Quevedo and Michael P. Ryan, Jr \\ Instituto de Ciencias Nucleares, \\ UNAM, A. Postal 70-543,$$
\text { México } 04510 \text { D.F., Mexico }
$$

\begin{abstract}
We consider three methods by which one can generate new cosmological models. Two of these are based on the Lorentzian structure of spacetime. In a Lorentzian manifold there can exist horizons that separate regions of spacetime that can be interpreted as cosmological models from others that have the character of "black holes." A number of well known solutions of this type can be used to generate both known cosmological models and others that do not seem to have been recognized. Another method based on the Lorentzian character of spacetime is to simply interchange some space variable with time and try to restructure the metric to make a viable cosmology.

A more broad-ranging method is the use of modern solution-generating techniques to construct new models. This method has been widely used to generate black hole solutions, but seems not to have been so widely used in cosmology. We will discuss examples of all three methods.
\end{abstract}

\footnotetext{
*Published in: "Mathematical and Quantum Aspects of Relativity and Cosmology", S. Cotsakis and G. W. Gibbons (Eds.) (Springer Verlag, Berlin, 2000) pp. 191-213

†Electronic address: quevedo, ryan@nuclecu.unam.mx
} 


\section{INTRODUCTION}

The standard model of cosmology assumes that the universe today is homogeneous and isotropic, which means that there are six Killing vectors of the manifold that give the isometries that realize these symmetries. However, in earlier stages of the universe it is assumed that there might have been large amounts of anisotropy and inhomogeneity that, by some physical process, could have been reduced to the point where today we observe the standard model (for a compendium of reasons for considering inhomogeneous models,

see Ref. [1]). Anisotropic and inhomogeneous metrics have fewer and fewer Killing vectors, and we could, in principle, arrive at a completely general solution of the Einstein equations as a cosmological model. At some point it will become difficult to distinguish between a cosmological model and any other general solution of the Einstein equations, except as a matter of interpretation. In fact, even an inhomogeneous cosmology with two spacelike Killing vectors is difficult to distinguish (locally) from gravitational waves propagating in one space direction. One method that has been used to separate cosmological models from pure wave solutions is the prescription of their global topology. If we insist that cosmologies have compact $t=$ constant surfaces, then a number of gravitational wave solutions can be made into cosmologies by compactifying in certain directions of the system. We will give examples of this procedure below. If we allow open universes, whether we call a solution a cosmological model or not is again a matter of interpretation.

With these caveats we can give a number of methods by means of which one can transform known solutions of the Einstein equations into cosmological models. These methods can be broken down into three broad classes:

1) Horizon methods

2) Causal structure methods

3) Mapping methods

The first of these methods has a long history, even though it never seems to have been thought of as a "method." Perhaps the oldest example is the deSitter metric 2], although it is a somewhat degenerate example. Originally deSitter proposed a solution to the Einstein equations with cosmological constant $\Lambda$ of the form [ $[3]$ 


$$
d s^{2}=-\left(1-\frac{\Lambda r^{2}}{3}\right) d t^{2}+\frac{d r^{2}}{\left(1-\frac{\Lambda r^{2}}{3}\right)}+r^{2}\left(d \theta^{2}+\sin ^{2} \theta d \varphi^{2}\right) .
$$

This seems to be a static metric similar in form to the Schwarzschild metric, and even has a "singularity" (which caused much comment at the time) at $r=\sqrt{3 / \Lambda}$. Of course, this singularity is just a horizon where the light cones tip over sufficiently that for $r>\sqrt{3 / \Lambda}$, $\partial / \partial r$ becomes timelike and $\partial / \partial t$ spacelike. For large $r$, then, we can rename the coordinates $(r \rightarrow \tilde{t}, t \rightarrow \tilde{r})$, and the new metric is

$$
d s^{2}=-\frac{d \tilde{t}^{2}}{\frac{\Lambda \tilde{t}^{2}}{3}-1}+\left(\frac{\Lambda \tilde{t}^{2}}{3}-1\right) d \tilde{r}^{2}+\tilde{t}^{2}\left(d \theta^{2}+\sin ^{2} \theta d \varphi^{2}\right),
$$

which is an obvious cosmological model. It was "recognized" (the reason for using the quotation marks will become obvious below) relatively quickly that this was indeed a cosmological model by making use of the coordinate transformation

$$
\begin{gathered}
\tilde{t}=r e^{\sqrt{\Lambda / 3} T}, \quad \tilde{r}=T-\frac{1}{2} \sqrt{\frac{3}{\Lambda}} \ln \left(\frac{\Lambda r^{2} e^{2 \sqrt{\Lambda / 3} T}}{3}-1\right), \\
r \sin \theta \cos \varphi=X, \quad r \sin \theta \sin \varphi=Y, \quad r \cos \theta=Z,
\end{gathered}
$$

which makes the metric

$$
d s^{2}=-d T^{2}+e^{2 \sqrt{\Lambda / 3} T}\left[d X^{2}+d Y^{2}+d Z^{2}\right]
$$

which is a $k=0$ Friedmann-Robertson-Walker metric.

Here we should study the Penrose diagram of this metric. In Figure 1 we give a Penrose diagram that will cover several cases that we will discuss later. Because of this we will give only generic values in the figure, and each case will correspond to different values of these parameters. In the deSitter case, the lines $a a$ and $b b$ correspond to $r=\sqrt{3 / \Lambda}$, and both $\pm I$ to $r=0$. In region I the spacelike Killing vector $\partial / \partial r$ generates spacelike surfaces that are the $t=$ constant surfaces of (11). In region II the same Killing vector is now $\partial / \partial \tilde{t}$ (timelike) and generates timelike hypersurfaces $\tilde{r}=$ constant. The horizon splits the region comprised by both regions I and II in two. Region I might be called a "black hole" region since the metric is reminiscent of the Schwarzschild metric, and has the spacelike Killing vector $\partial / \partial r$, while region II is a "cosmological" region with metric (4). In the cosmological region there seem to be "singularities" at $T= \pm \infty$, but we can see that $\left[\right.$ note that $T=\tilde{r}+\frac{1}{2} \sqrt{\frac{3}{\Lambda}} \ln \left(\frac{\Lambda \tilde{t}^{2}}{3}-1\right)$ ] 


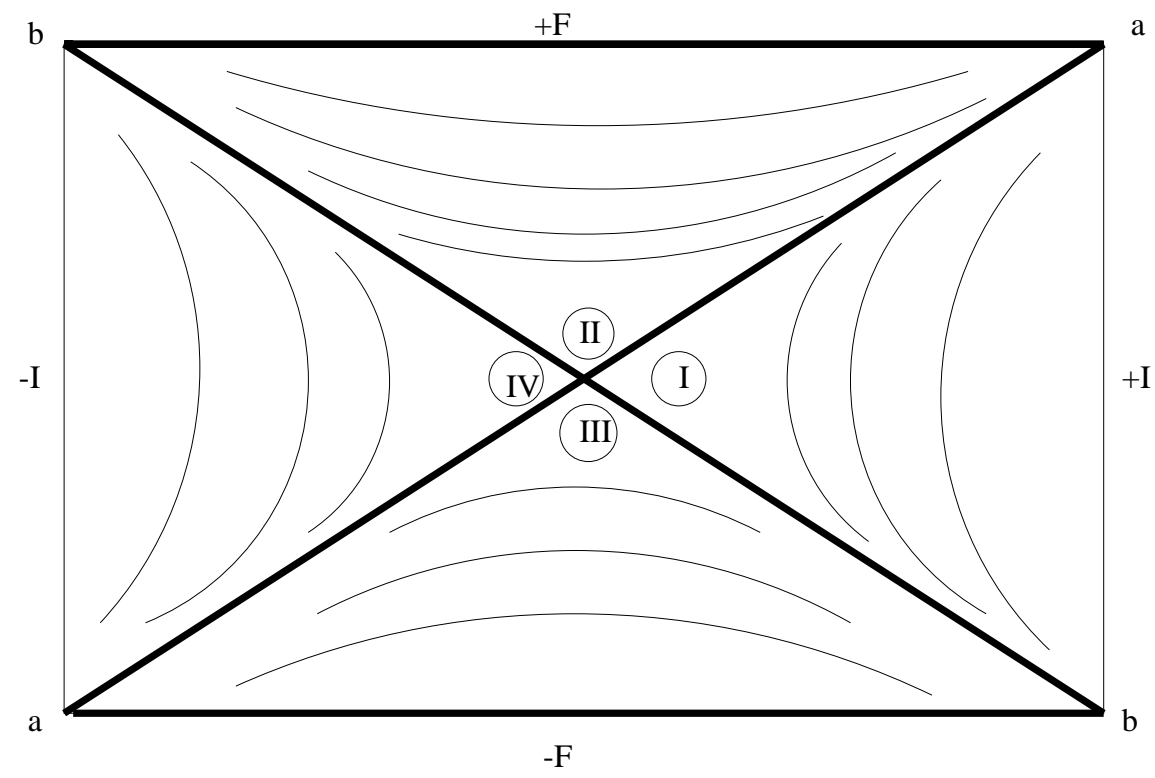

FIG. 1: A generic Penrose diagram which will be used for several metrics of the article

the singularity at $T=-\infty$ is just the horizon at $\tilde{t}=\sqrt{3 / \Lambda}$, so it is nothing more than a null surface separating two parts of the spacetime.

The problem here is that the deSitter metric has such a high degree of symmetry (a space of constant curvature with 10 Killing vectors) that the distinction between regions I and II is artificial. In fact, the coordinate transformation (3) (with $\tilde{t} \rightarrow r$ and $\tilde{r} \rightarrow t$ and $\frac{\Lambda \tilde{t}^{2}}{3}-1 \rightarrow \frac{1-\Lambda r^{2}}{3}$ ) is equally valid in region $\mathrm{I}$, and, in fact, this was what was recognized by Lemaitre [4] and Robertson [5] in transforming the deSitter metric into the form that was eventually used in the steady-state model. Actually, even Minkowski space (also a space of constant curvature with 10 Killing vectors) can be looked at in the same way, as we will see in Sec. 2.

In Sec. 2 we will consider several metrics where horizon methods can be used to construct cosmological models that do not have the disadvantages of the deSitter metric, that is, there exists no coordinate transformation that is valid for the static region which transforms it into a cosmological model. The fact that "singularities" in the new cosmological models may just be null surfaces where one passes from one coordinate patch to another, will, of course, still be a feature, although true curvature singularities may also exist. One point that should be mentioned is that most of the models generated by this method that we will consider are vacuum models. There are some electrovac models that can be thought of as 
being generated by horizon methods that we will mention, but it seems not to be known which matter-filled cosmological models can be generated by this method.

The second class of methods, causal structure methods, is similar to the previous one, but there is no horizon to distinguish different regions of spacetime. The paradigm of this method is the unpolarized Gowdy $T^{3}$ vacuum spacetime [6] which will be discussed in more detail below. The idea behind this method is to begin with a known solution and simply rename some spacelike variable as $t$, and the timelike variable as a spacelike variable (we can call this $t \leftrightarrow r$ ), and then change the causal structure of the resulting metric so that the a vector tangent to the "time" direction is truly timelike, and one tangent to the new "space" direction is spacelike. There are a number of problems with this approach. The first is that determining the causal structure change is not necessarily trivial. In a diagonal metric this difficulty can usually be handled by simply changing the signs of two of the metric components, but in a more general metric the procedure may be more complicated. The most important difficulty, however, is that the new metric is not guaranteed to be a solution to the Einstein equations. For matter filled models, where physical quantities can be used to define spacelike and timelike surfaces, the new metric will almost certainly not be a solution. For vacuum metrics it is difficult to tell. As with horizon methods, the known solutions of this type are vacuum wave solutions, and they have rather simple structures, and give inhomogeneous cosmological models. Here, of course, one has the problem of distinguishing between gravitational wave solutions and inhomogeneous models.

A very simple example of this procedure is the plane wave []

$$
d s^{2}=L^{2}(u)\left(e^{2 \beta(u)} d x^{2}+e^{-2 \beta(u)} d y^{2}\right)+d z^{2}-d t^{2},
$$

where $u=t-z$. If we make the coordinate change $t \rightarrow \tilde{z}, z \rightarrow \tilde{t}$ and change the signs of $d z^{2}$ and $d t^{2}$, we find $(\tilde{u}=\tilde{t}-\tilde{z})$

$$
d s^{2}=L^{2}(-\tilde{u})\left[e^{2 \beta(-\tilde{u})} d x^{2}+e^{-2 \beta(-\tilde{u})} d y^{2}\right]-d \tilde{t}^{2}+d \tilde{z}^{2}
$$

Since $L$ and $\beta$ are arbitrary function of their arguments, we can remove the minus signs in the arguments of $L$ and $\beta$ in (6) and obtain (5) again. Because of the high symmetry of the problem, this procedure gives the same metric and Einstein equations, and there is no question that any solution of the Einstein equations for (5) gives a solution to (6) . Here, of course, one runs into the problem of defining a cosmological model. Is either of (5) 
or (66) a gravitational wave or an inhomogeneous cosmological model? Either one is both, depending on how we interpret them. If, however, we insist that a cosmological model have compact $t=$ const. sections, then we can compactify (6) in the space directions by just making $0 \leq x, y, z \leq 2 \pi$ and identifying the points at 0 and $2 \pi$, giving the manifold a $T^{3}$ topology. This changes the boundary conditions on the functions $L$ and $\beta$ in that one must have $L(t, 0)=L(t, 2 \pi)$ and $\beta(t, 0)=\beta(t, 2 \pi)$. Since the only Einstein equation is

$$
L^{\prime \prime}(\tilde{u})+\left[\beta^{\prime}(\tilde{u})^{2}\right] L=0
$$

$'=d / d \tilde{u}, \beta$ is arbitrary except for the boundary conditions, and $L$ obeys a simple equation that has solutions that satisfy the boundary conditions. Here one must be careful not to change coordinates back to the original metric, since we will generate a spacetime with closed timelike lines, a problem with cosmological models generated in this manner. We will discuss examples and their topology in Sec. 2.

The third method consists of taking known solutions and mapping them to new solutions. This technique is similar in spirit to conformal mapping. Any analytic function of one complex variable, $w=f(z)$ maps $z=x+i y$ to $w=u(x, y)+i v(x, y)$, where, if $\psi(x, y)$ is a solution to Laplace's equation, $\Psi[u(x, y), v(x, y)]$ is also a solution. This idea exploits the invariance of Laplace's equation in two dimensions under the two-dimensional conformal group. If one can find groups under which the Einstein equations are invariant (or under which some class of Einstein equations for special systems are invariant), one can generate new solutions from old. This technique is of wide applicability, and has been exploited heavily in general relativity to find new exact solutions. It is by no means specific to cosmological models, but it can be applied in cosmological situations, and has, perhaps, been underutilized in this field and deserves more attention there.

In the specific case of stationary axisymmetric spacetimes, early generating methods such as the Kerr-Schild ansatz [8, 9, 10, 11], the complex transformation method 12, 13, 14], and Hamilton-Jacobi separability [15] were used to derive new solutions, but all of them are contained in the charged Kerr-Taub-NUT [16] class (with cosmological constant). An important development for the derivation of exact stationary axisymmetric solutions was made by Ernst [17, 18], who obtained a new representation of the corresponding field equations which is independent of the coordinates chosen and, therefore, allows one to investigate the symmetries involved and find new solutions. Ernst also proposed two generating techniques 
that were later enlarged and unified by Kinnersley [19, 20]. Using the compact Ernst formulation of the field equations it was also possible to obtain new solutions [21, 22, 23, 24, 25] possessing a certain prescribed polynomial form of the Ernst potential. Modern solution generating techniques involve Lie groups of transformations or Bäcklund transformations. The first such group was found by Geroch [26, 27] and generalized by Cosgrove [28, 29]. The Geroch group was investigated very intensively and it was found that it possesses subgroups that preserve asymptotic flatness [19, 30, 31, 32, 33]. Today, there exist three main solution generating tecniques: HKX (Hoenselaers, Kinnersley, Xanthopoulos) transformations

34, 35], which are based upon special subgroups of the Geroch group, Bäcklund transformations [36, 37], which are applied directly to the Ernst potential, and the inverse scattering method [38, 39], which is based on a reformulation of the nonlinear field equations as a linear eigenvalue problem. Later on it was shown [40, 41] that all these techniques are related to one another, and can be used to generate the same type of solutions (for an introductory review of the solution generating techniques and the relationships between them see [42]).

The mapping method is by no means specific to stationary axisymmetric metrics, but it can be applied to any spacetime characterized by two or more commuting Killing vector fields as in the case of the cosmological models investigated in this work. We will give some examples in Sec. 3 and a guide to possible new uses of the technique.

The paper is organized as follows. Section 2 will give examples of horizon and causal structure methods and solutions obtained using them. Section 3 will give one example of mapping methods and discuss others. Finally, Section 4 will discuss possible new directions in the use of known solutions to find new cosmologies.

\section{HORIZON AND CAUSAL STRUCTURE METHODS}

\section{A. Horizon Methods}

There are a large number of solutions of the Einstein equations that have cosmological coordinate patches as well as "black hole" regions that are separated by horizons. The major problem with the cosmologies generated in this way is they are incomplete manifolds, and what have in the past been interpreted in some of them as singularities are just the horizons that separate one part of the complete manifold from the other. A second problem, as we 
have seen, is that in certain cases the complete manifold is of such high symmetry that is is impossible to distinguish between the cosmological region and the "black hole" region. The most blatant example of the second problem is just ordinary Minkowski space.

If we return to Figure 1, we can think of this diagram as a picture of Minkowski space with the meeting point of lines $a a$ and $b b$ an arbitrarily chosen origin $O$. The usual metric is

$$
d s^{2}=-d T^{2}+d x^{2}+d y^{2}+d z^{2} .
$$

In region I the hyperbolic lines are just the usual orbits of a particle with constant acceleration that form the basis of Rindler [43] space, and if the lines are given by $x=\sqrt{2 r-1} \cosh t$ and $T=\sqrt{2 r-1} \sinh t, z=y=$ const., the metric becomes

$$
d s^{2}=-(2 r-1) d t^{2}+\frac{d r^{2}}{2 r-1}+d y^{2}+d z^{2} .
$$

In this coordinate system the metric is very similar in form to the Schwarzschild metic, and there is a coordinate singularity at $r=1 / 2$, which is a horizon similar to that of the deSitter metric.

In Figure 1 the line $b b$ is the surface $r=1 / 2$, and for $r<1 / 2$ we can make the $t \leftrightarrow r$ coordinate change and the metric becomes

$$
d s^{2}=-\frac{1}{1-2 t} d t^{2}+(1-2 t) d r^{2}+d y^{2}+d z^{2},
$$

a cosmological model. If we change coordinates using $t=\frac{1}{2}\left[1-\tau^{2}\left\{\cosh ^{2} \rho-\right.\right.$ $\left.\left.\sinh ^{2} \rho \sin ^{2} \theta \cos ^{2} \varphi\right\}\right], r=\tanh ^{-1}[\tanh \rho \sin \theta \cos \varphi], y=\tau \sinh \rho \sin \theta \sin \varphi, z=\tau \sinh \rho \cos \theta$, the metric (10) becomes the Milne universe, a flat cosmological model with metric

$$
d s^{2}=-d \tau^{2}+\tau^{2}\left[d \rho^{2}+\sinh ^{2} \rho\left(d \theta^{2}+\sin ^{2} \theta d \varphi^{2}\right)\right],
$$

a $k=-1$ FRW metric. Of course, the manifold is nothing more than flat space and the breaking up of the manifold into regions I-IV is an observer-dependent phenomenon due to singling out the origin $O$ as the position of a special observer, while there is actually no physical reason that this point is priveleged over any other point of the manifold, and there is no real difference between any of the four regions. Basically, we can say that the same is true of deSitter space, which is just a constant curvature space everywhere, and the "cosmological" region has nothing to distinguish it from any other region of the manifold. 
There are a number of metrics which are not as degenerate as the Minkow- ski and deSitter cases whose cosmological regions are physically distinguishable from the "black hole" regions, and the resulting cosmological models have long been known and are named. Perhaps the simplest of these is the Kantowski-Sachs-Schwarzschild manifold. Here we can still use the generic Figure 1 to represent the Penrose diagram of this metric, with region I representing the Schwarzschild coordinate patch where the hyperbolic lines are $r=$ const. lines and the lines $b b$ is the horizon at $r=2 m$. In region II the hyperbolic lines are still $r=$ const. lines, but they no longer represent spacelike surfaces. For $r>2 m$ the metric can be written as

$$
d s^{2}=-\left(1-\frac{2 m}{r}\right) d t^{2}+\frac{1}{1-\frac{2 m}{r}} d r^{2}+r^{2}\left(d \theta^{2}+\sin ^{2} \theta d \varphi^{2}\right),
$$

but for $r<2 m$ the Killing vector $\partial / \partial r$ is no longer spacelike, and we may make the transformation $r \leftrightarrow t$ mentioned in Sec. 1, and we find

$$
d s^{2}=-\frac{1}{\frac{2 m}{t}-1} d t^{2}+\left(\frac{2 m}{t}-1\right) d r^{2}+t^{2}\left(d \theta^{2}+\sin ^{2} \theta d \varphi^{2}\right),
$$

which is an obvious cosmological model, the vacuum Kantowski-Sachs model. The fact that at $r=0$ ( $t=0$ in the new coordinate system) which is represented by the line at $+F$ is a true curvature singularity distinguishes region II from region I and makes it impossible to find a global coordinate transformation that makes this cosmological model indistinguishable from the "black hole" region. Of course, however, this cosmological model, and all cosmological models generated by horizon methods, have the problem that what has been regarded as singularities in these models may not be a curvature singularity, but only a horizon where we pass from one coordinate patch to another. In the case of the Kantowski-Sachs solution the $t=0$ singularity is one of the usual singularities of the model, and it is a true curvature singularity. The other "singularity" at $t=2 m$ is only a null surface.

While the Kantowski-Sachs solution, even though it was originally discovered by means of studies of groups of motion that were not transitive on three surfaces, and constituted a generalization of the Bianchi cosmological models, and was immediately recognized as the Schwarzschild solution inside the horizon, in the next model we will discuss the two regions on either side of the horizon were studied separately. The "black hole" region was the NUT space of Newman, Tamburino and Unti 9], and the cosmological region was the Taub cosmology [4]. Misner [45] showed that these two solutions could be seen to be part of a larger manifold (up to topological questions which will be discussed below). In the black 
hole region one can write the NUT metric as [46]

$$
\begin{array}{r}
d s^{2}=-\frac{r^{2}-2 m r-l^{2}}{r^{2}+l^{2}}(d t+2 l \cos \theta d \varphi)^{2}+\frac{r^{2}+l^{2}}{r^{2}-2 m r-l^{2}} d r^{2} \\
+\left(r^{2}+l^{2}\right)\left(d \theta^{2}+\sin ^{2} \theta d \varphi^{2}\right) .
\end{array}
$$

For the region of this metric where $0<r<m\left(1+\sqrt{1+l^{2} / m^{2}}\right)$, the $r \leftrightarrow t$ transformation gives

$$
\begin{array}{r}
d s^{2}=\frac{l^{2}+2 m t-t^{2}}{t^{2}+l^{2}}(d r+2 l \cos \theta d \varphi)^{2}-\frac{t^{2}+l^{2}}{l^{2}+2 m t-t^{2}} d t^{2} \\
+\left(t^{2}+l^{2}\right)\left(d \theta^{2}+\sin ^{2} \theta d \varphi^{2}\right),
\end{array}
$$

which is an anisotropic cosmological model. The major problem with this model is that in the cosmological sector we can rewrite the metric on $t=$ const. surfaces by making the coordinate transformation

$$
\begin{aligned}
& y=\theta-\pi / 2, \\
& z=r / 2 l, \\
& x=\varphi
\end{aligned}
$$

which gives the three-dimensional line element as

$$
d \sigma^{2}=\frac{4 l^{2}\left(l^{2}+2 m t-t^{2}\right)}{t^{2}+l^{2}}(d z-\sin y d x)^{2}+\left(t^{2}+l^{2}\right)\left(d y^{2}+\cos ^{2} y d x^{2}\right)
$$

which can be written as

$$
d \sigma^{2}=\frac{4 l^{2}\left(l^{2}+2 m t-t^{2}\right)}{t^{2}+l^{2}}\left(\omega^{3}\right)^{2}+\left(t^{2}+l^{2}\right)\left[\left(\omega^{1}\right)^{2}+\left(\omega^{2}\right)^{2}\right]
$$

where $\omega^{3}=d z-\sin y d x, \omega^{1}=\cos y \cos z d x-\sin z d y, \omega^{2}=\cos y \sin z d x+\cos z d y$. These differential forms are the invariant one-forms of the Bianchi type IX cosmological models, the invariant one-forms on $S^{3}$. This means that the "natural" topology of the three-surfaces given by (20) is that of $S^{3}$, with $0 \leq z \leq 4 \pi, 0 \leq x \leq 2 \pi, 0 \leq y \leq \pi$. If one maintains this topology on passing through the horizon, the vector $\partial / \partial z$ becomes timelike, and the $S^{3}$ topology implies the possibility of closed timelike lines. This topological difficulty is present in many cosmological models generated by horizon methods, as we will see below.

The metrics we have discussed seem to be the only ones where the cosmological models have been noticed explicitly to be a part of a larger manifold that has non-cosmological 
sectors. For example, it is only recently that the Kerr metric inside its horizon has been considered as a cosmological model. Of course, the metric in this part of the Kerr manifold has been studied [47], but there seems to have been no attempt to identify it as a cosmological model. In Boyer-Lindquist coordinates the Kerr metric has the form

$$
\begin{array}{r}
d s^{2}=-\frac{r^{2}-2 M r+a^{2}}{r^{2}+a^{2} \cos ^{2} \theta}\left[d t-a \sin ^{2} \theta d \phi\right]^{2}+\frac{\sin ^{2} \theta}{r^{2}+a^{2} \cos ^{2} \theta}\left[\left(r^{2}+a^{2}\right) d \phi-a d t\right]^{2} \\
+\frac{r^{2}+a^{2} \cos ^{2} \theta}{r^{2}-2 M r+a^{2}} d r^{2}+\left(r^{2}+a^{2} \cos ^{2} \theta\right) d \theta^{2}
\end{array}
$$

This metric has two horizons, $r_{ \pm}=M \pm \sqrt{M^{2}-a^{2}}$, and beyond the outer horizon, $r_{+}, \partial / \partial r$ is a spacelike vector, and the metric represents a spinning black hole. At $r_{+}$the light cones tip over to the point where $\partial / \partial r$ becomes timelike and we can make the transformation $t \leftrightarrow r$. Unfortunately, at the inner horizon $r_{-}$the light cones tip back to the point where $\partial / \partial r$ becomes spacelike again. In the region $r_{-}<r<r_{+}$the Kerr metric becomes a cosmological model,

$$
\begin{array}{r}
d s^{2}=\frac{2 M t-t^{2}-a^{2}}{t^{2}+a^{2} \cos ^{2} \theta}\left[d r-a \sin ^{2} \theta d \phi\right]^{2}+\frac{\sin ^{2} \theta}{t^{2}+a^{2} \cos ^{2} \theta}\left[\left(t^{2}+a^{2}\right) d \phi-a d r\right]^{2} \\
-\frac{t^{2}+a^{2} \cos ^{2} \theta}{2 M t-t^{2}-a^{2}} d t^{2}+\left(t^{2}+a^{2} \cos ^{2} \theta\right) d \theta^{2} .
\end{array}
$$

This metric, with the simple transformation [48],

$$
t=\alpha\left[\sqrt{1-\beta^{2}} \cos \left(e^{-\tau}\right)+1\right]
$$

$\alpha=M, \beta=a / M(0 \leq \beta \leq 1)$ transforms (22) into

$$
\begin{array}{r}
d s^{2}=e^{-\lambda / 2} e^{\tau / 2}\left(-e^{-2 \tau} d \tau^{2}+d \theta^{2}\right)+\alpha \sqrt{1-\beta^{2}} \sin \left(e^{-\tau}\right)\left[e^{P} d \delta^{2}\right. \\
\left.+2 e^{P} Q d \delta d \phi+\left(e^{P} Q^{2}+e^{-P} \sin ^{2} \theta\right) d \phi^{2}\right]
\end{array}
$$

where

$$
\begin{gathered}
\lambda=\tau-2 \ln \left(\alpha^{2}\left\{\left[\sqrt{1-\beta^{2}} \cos \left(e^{-\tau}\right)+1\right]^{2}+\beta^{2} \cos ^{2} \theta\right\}\right), \\
P=\ln \left[\left(1-\beta^{2}\right) \sin ^{2}\left(e^{-\tau}\right)+\beta^{2} \sin ^{2} \theta\right]-\ln \left[\alpha \sqrt{1-\beta^{2}} \sin \left(e^{-\tau}\right)\right] \\
-\ln \left(\left[\sqrt{1-\beta^{2}} \cos \left(e^{-\tau}\right)+1\right]^{2}+\beta^{2} \cos ^{2} \theta\right), \\
Q=-\frac{2 \alpha \beta \sin ^{2} \theta\left[\sqrt{1-\beta^{2}} \cos \left(e^{-\tau}\right)+1\right]}{\left(1-\beta^{2}\right) \sin ^{2}\left(e^{-\tau}\right)+\beta^{2} \sin ^{2} \theta} .
\end{gathered}
$$

The metric (24) is a Gowdy cosmological model with $S^{1} \times S^{2}$ topology []. . 

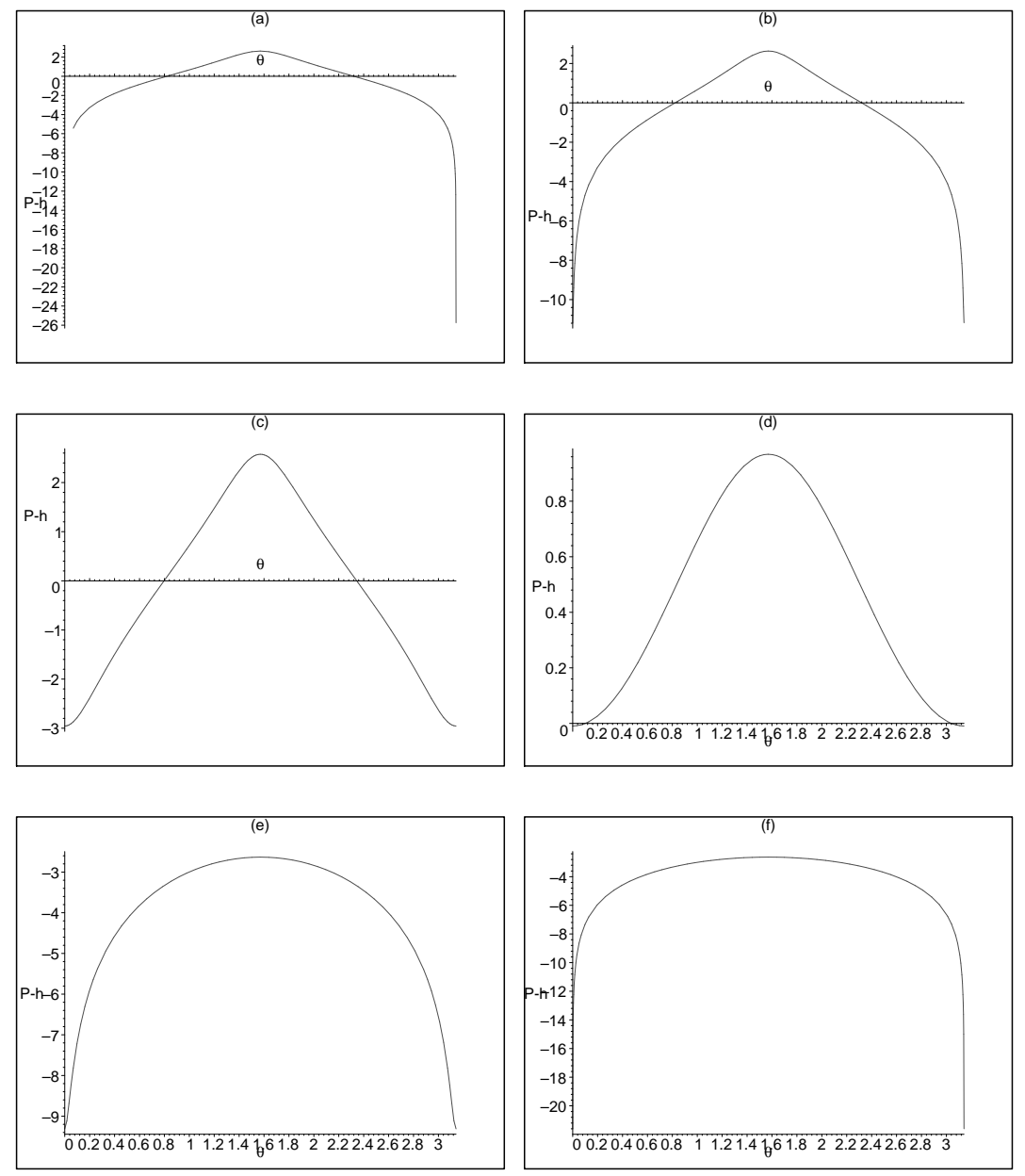

FIG. 2: The evolution in $\tau$ of $P-h$ as a function of $\theta$ from Eqs. (25,27) for $\alpha=1, \beta=1 / 2$. Fig. (a) corresponds to $\tau=-\ln (\pi)$, (b) to $\tau=-1.144$, (c) to $\tau=-1.1$, (d) to $\tau=-0.75$, (e) to $\tau=+5$, (f) to $\tau=+10$

The solution given in Eqs. (25] 227) is a function of $\theta$ for $0 \leq \theta \leq \pi$ and is valid for values of $\tau$ that correspond to the part of the manifold between $r_{-}$and $r_{+}$, that is, for $-\ln (\pi) \leq \tau \leq+\infty$, note that $t$ takes the values $M \pm \sqrt{M^{2}-a^{2}}$ at the two limiting values of $\tau)$.

As an example, Figs. 22 and 3 give $P-h\left(h=-\ln \left[\alpha \sqrt{1-\beta^{2}} \sin \left(e^{-\tau}\right)\right]\right)$ and $Q$ as functions of $\theta$ for various values of $\tau$ for $\alpha=1, \beta=1 / 2$. The evolution of these two functions is that of a "spike" that at the limiting values of $\tau$ is practically flat (except at $\theta=0, \pi$, where it drops off drastically), and which becomes sharper for intermediate values of $\tau$. It should be mentioned that the Kerr metric is a special case of what is usually called the Kerr-Taub- 

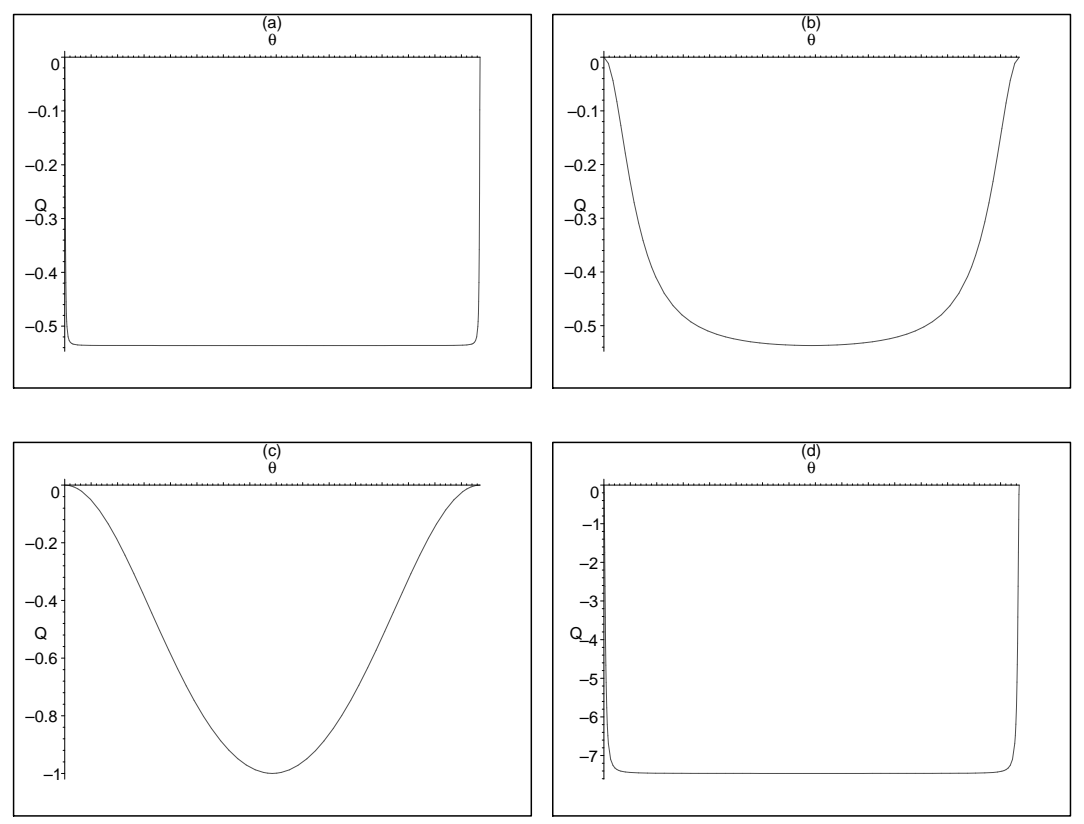

FIG. 3: The evolution of $Q$ as a function of $\theta$ in $\tau$. Fig. (a) corresponds to $\tau=-1.1439$, (b) to $\tau=-1.1,(\mathrm{c})$ to $\tau=-\ln (\pi / 2),(\mathrm{d})$ to $\tau=+5$

NUT metric, which is geven in Ref. [46]. This metric has three parameters, $a, m, l$, the Kerr parameter, the mass, and the NUT parameter respectively. For $a=0$ we have the metric (14), and for $l=0$ we have the Kerr metric. This means that the Gowdy model we have given is also a special case of the "Taub" region of Kerr-Taub-NUT. However, the Taub region of this manifold, in spite of its name, never seems to have been considered as a cosmological model.

As we have stated several times, the models studied so far are vacuum models. While matter-filled models may, in principle, be generated by horizon methods, in at least some cases the models with matter have very different behavior from the vacuum case, and may preclude naive generalizations of the method to the non-vacuum case. For instance, it is known that the Taub model with fluid matter has true curvature singularities where the vacuum case has null surfaces [49]. The same is true for the Kantowski-Sachs model [50]. However, the Brill model [51] is a Taub-NUT electrovac solution which is non-singular in the same way as the Taub-NUT vacuum case. In fact, the Reissner-Nordstrøm solution with electric charge large enough has two horizons, and between the outer and inner horizons there is a region that can be interpreted as an electrovac cosmology that has no curvature 
or electromagnetic field singularity at the horizons.

Besides the solutions we have mentioned, there are numerous metrics that represent gravitational fields outside some material body which in vacuum have "black hole" sectors outside of some horizon. Of course, no hair theorems [52] say that there are no true vacuum black holes that are not Kerr or Schwarzschild. Solutions of the type mentioned above seem always to have curvature singularities on or outside their horizons where they can be seen by observers at infinity. While this fact makes them poor candidates for black holes, it makes them interesting as cosmological models inside their horizons. The "singularities" of the models with curvature singularities on their horizons will be inhomogeneous, with part of the singularities being simple horizons, but other regions will be true curvature singularities. These structures might be of great interest in the study of cosmological singularities. Perhaps the simplest of the metrics of this type might be the Tomimatsu-Sato [21, 22] models which do have inhomogenous horizons, but there are other known solutions, for example, one with many multipoles and large curvature singularity regions on the horizon [53].

\section{B. Causal Structure Methods}

This method seems to have been little used, and perhaps the most telling reason for this is that there are very few examples where it works. The only example that has wide currency is that of certain Gowdy models. If one begins with the Einstein-Rosen waves [54], which have the metric

$$
d s^{2}=e^{2 \gamma-2 \psi}\left(d r^{2}-d t^{2}\right)+r^{2} e^{-2 \psi} d \phi^{2}+e^{2 \psi} d z^{2}
$$

with $\gamma$ and $\psi$ functions of $r$ and $t$, the Einstein equations for this metric are

$$
\begin{gathered}
\psi_{, r r}+\frac{1}{r} \psi_{, r}-\psi_{, t t}=0 \\
\gamma_{, r}=r\left(\psi_{, r}^{2}+\psi_{, t}^{2}\right), \quad \gamma_{, t}=2 r \psi_{, r} \psi_{, t},
\end{gathered}
$$

where it is well known that the equations for $\gamma$ can be integrated directly once $\psi$ is known, since the integrability condition for these two first-order partial differential equations is just (29).

If we now simply make the coordinate change $t \leftrightarrow r$, we have

$$
d s^{2}=e^{2 \gamma-2 \psi}\left(d t^{2}-d r^{2}\right)+t^{2} e^{-2 \psi} d \phi^{2}+e^{2 \psi} d z^{2}
$$


Here the only obstacle to interpreting this metric as a cosmological model is the wrong signs in the $d r^{2}$ and $d t^{2}$ terms if we want to interpret $\partial / \partial r$ and $\partial / \partial t$ as spacelike and timelike vectors respectively. If we add a complex constant to $\gamma, \gamma=\tilde{\gamma}+i \pi$, we change the signs of $g_{t t}$ and $g_{r r}$, and since we have done nothing more than change the names of $r$ and $t$, the Einstein equations are unchanged (since they depend only on derivatives of $\gamma$ ), and we have

$$
\begin{gathered}
-\psi_{, t t}-\frac{1}{t} \psi_{, t}+\psi_{, r r}=0 \\
\tilde{\gamma}_{, t}=t\left(\psi_{, t}^{2}+\psi_{, r}^{2}\right), \quad \tilde{\gamma}_{, r}=2 t \psi_{, t} \psi_{, r}
\end{gathered}
$$

These are the original equations for the unpolarized Gowdy $T^{3}$ cosmological model [6].

Notice that in order to change the sign of the $d t^{2}-d r^{2}$ term, it was necessary to add $-i \pi$ to $\gamma$, and if the Einstein equations were to depend on $\gamma$ in any other way than through derivatives or $e^{\gamma}$, they would become complex and then would not necessarily have real solutions for $\gamma$ and $\psi$. This difficulty is a paradigm for the problems one would encounter in trying to use a $t \leftrightarrow r$ type of coordinate change. One would not expect this technique to be successful in the great majority of cases, especially if there were matter present. This seems to be reflected in the fact that there exist few examples of this method. Note that the examples we have given, the plane wave of the Introduction and the $T^{3}$ Gowdy model, are vacuum spacetimes, where the temporal variable and one of the space variables appear in the form of $-d t^{2}+d z^{2}$ (in the Gowdy model, multiplied by a conformal factor). While we will not try to explore this kind of idea further here, perhaps theorems about the existence of solutions found by means of the method could be based on this fact.

\section{Topological Questions}

An interesting feature of cosmological models generated by means of horizon and causal structure methods is that for philosophical reasons one often wants these models to have compact $t=$ const. sections, that is, they should be closed cosmological models. This can be achieved for many of the solutions given above by simply specifying the global topology without changing the local geometry. This has caused some problems with these models, especially the vacuum models generated by horizon methods, since they represent incomplete manifolds, and when one passes through the horizon, the spacelike direction in which the 
manifold would have to be closed becomes timelike, leading to the possibility of closed timelike lines in the "black hole" sector.

Perhaps the simplest model where this occurs is the Kantowski-Sachs- Schwarzschild manifold. If we use (13) for the Kantowski-Sachs model, notice that the $\theta \varphi$ sector has a natural two-sphere topology, and there is no obstruction to compactifying in the $r$ direction by simply assuming that $0 \leq r \leq 2 \pi$, with $r=0$ and $r=2 \pi$ identified, giving the manifold an $S^{1} \times S^{2}$ topology. Of course, if we consider the other side of the horizon, the $t \leftrightarrow r$ reparametrization means that the $r$-direction becomes the $t$-direction, and we have $0 \leq t \leq 2 \pi$ and the possibility of closed timelike lines. In principle one could have timelike lines which pass from the cosmological region to the black hole region and remain trapped in that region in an eternal closed timelike curve. In the case of the KantowskiSachs-Schwarzschild manifold it seems to be impossible for this to happen. From Fig. 4 one can see that timelike geodesics which begin inside the horizon will always stay within the horizon, simply passing through the crossing point of the past and future horizons (a focussing point for all geodesics) [55]. The choice of topology of this metric has a long history. The original studies of this metric assumed that $r$ ran from $-\infty$ to $+\infty$ and that the topology was $R^{1} \times S^{2}$. This topology was usually used until Laflamme and Shellard introduced the $S^{1} \times S^{2}$ topology 56]. Since that time most authors have used it, but some still prefer the $R^{1} \times S^{2}$ topology [57].

Probably the first solution where topological problems were noticed was the Taub-NUT model, where the natural topology of $\mathrm{t}=$ const. slices in the Taub model is $S^{3}$, which means that the same topology must apply to the $t \theta \varphi$ sector of NUT space. Here it is possible for timelike geodesics to leave the Taub region and pass into the NUT region, where they may be trapped in trajectories that return to the same spacetime point [45, 49, 58].

For the other solutions generated by horizon methods mentioned so far, it seems that we will have similar problems, but there has been no study of the effect of topology changes on the causal properties of the solutions. Even degenerate solutions such as the deSitter model and Minkowski space can be given different topologies by means of this type of identifications. Thus, for example, the Milne model, while it is locally flat, may be made into a more acceptable cosmology by means of such a global topology change, but it would suffer from the same problem as the other metrics of this type in that the Rindler space sector would then have an unacceptable topology. The Kerr-Gowdy solution has this same 


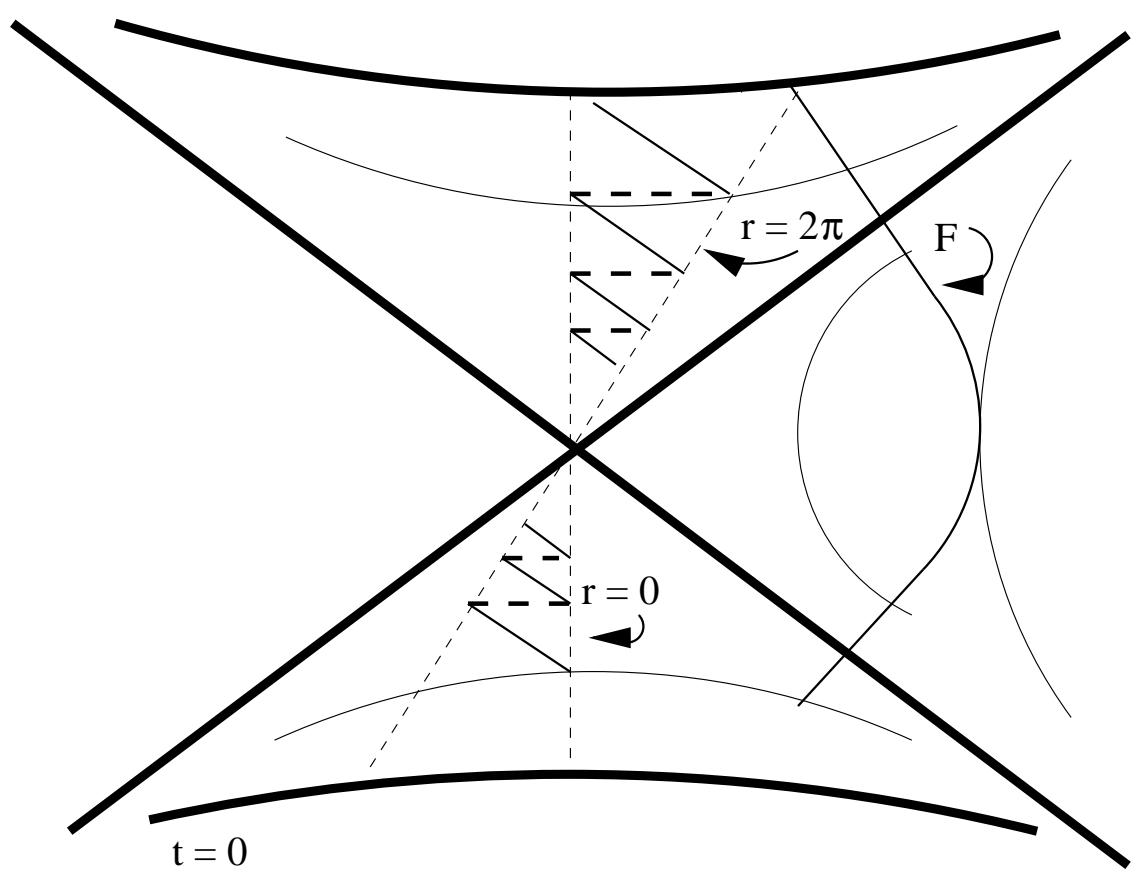

FIG. 4: A Kruskal diagram for geodesics in Kantowski-Sachs-Schwarzschild. For an open topology the timelike geodesic $F$ can leave the cosmological region, enter the Schwarzschild region and return to the cosmological region. This behavior is similar to that of some geodesics in Taub-NUT. For the closed topology, however, geodesics wrap around between $r=0$ and $r=2 \pi$ ( $r$ being the new "radial" direction inside the horizon), following the solid-dashed sawtooth path (here, as an example, for a lightlike geodesic), which always stays inside the horizon except at the crossing point of the past and future horizons

topological difficulty, since the interior is supposed to have an $S^{1} \times S^{2}$ topology, and outside the horizon this implies an $S^{1}$ topology in the timelike direction. The causal properties of this solution with this topology seem never to have been studied.

For metrics generated by causal structure methods, there is, in principle no obtruction to topology changes, since there is no "black hole" sector where new topologies can lead to causality problem. The Gowdy $T^{3}$ model is a good example of this. While we have not specified the ranges of $r, \phi$ and $z$ in (31), Gowdy, wishing to have a closed topology for the $t=$ const. surfaces in his model, assumed that $0 \leq r, \phi, z \leq 2 \pi$ with points at 0 and $2 \pi$ identified, which gives the model a three-torus topology. Since there is no "black hole" sector for the new manifold, there is no reason not to choose this topology. The only difference 
that this change introduces is in the boundary conditions that $\psi$ and $\gamma$ must satisfy. If $r$, $\phi$, and $z$ run from $-\infty$ to $+\infty$ the solutions of Eq. (32) are built up of eigenfunctions of continuous eigenvalues, while in the Gowdy topology the eigenvalues are discrete.

Note that if we were to try to make the $t \leftrightarrow r$ change for this topology, the EinsteinRosen wave thus generated would be closed in the time direction, but the Gowdy manifold is complete, and there is no reason to concern ourselves with this possibility.

As we mentioned in the Introduction, the somewhat artificial model we gave there may be closed in the space directions without changing the local metric.

\section{MAPPING METHODS}

As we mentioned in the Introduction, the solution generating tecniques developed in the last few decades have not often been used in the context of cosmological models. However, it is known that these techniques can be applied when the spacetime possesses at least two commuting Killing vector fields. This is exactly the case of the cosmological models under investigation in this paper.

In the original works on solution generating techniques the central idea was to project the spacetime on the two-dimensional hypersurface defined by the Killing vector fields. This projection allows one to reformulate the spacetime metric and the corresponding field equations in such a way that the symmetries of the differential equations can be investigated in a straightforward manner. An alternative but related procedure is to derive the Ernst representation of the field equations where it is possible to apply (with some modifications) known techniques. In the present work we will use this second alternative.

\section{A. Ernst Representation of the $T^{3}$ Gowdy Models}

In this section we will begin by considering the unpolarized $T^{3}$ Gowdy model. We will use a slightly different parametrization of these models from that used in (31) in order to make comparisons with Refs. [59]. The unpolarized model has the metric

$$
d s^{2}=e^{-\lambda / 2} e^{\tau / 2}\left(-e^{-2 \tau} d \tau^{2}+d \theta^{2}\right)+e^{-\tau}\left[e^{P}(d \sigma+Q d \delta)^{2}+e^{-P} d \delta^{2}\right],
$$

where the functions $\lambda, P$ and $Q$ depend on the coordinates $\tau$ and $\theta$ only, and $0 \leq \sigma, \delta, \theta \leq 2 \pi$. These spacetimes are characterized by the existence of two commuting Killing vector fields 
$\eta_{1}=\partial / \partial \sigma$ and $\eta_{2}=\partial / \partial \delta$. In the special case $Q=0$, the fields $\eta_{1}$ and $\eta_{2}$ become hypersurface orthogonal to each other and the metric (34) describes the polarized $T^{3}$ Gowdy models.

Einstein's vacuum field equations for the $T^{3}$ models consist of a set of two second order differential equations for $P$ and $Q$

$$
\begin{gathered}
P_{, \tau \tau}-e^{-2 \tau} P_{, \theta \theta}-e^{2 P}\left(Q_{, \tau}^{2}-e^{-2 \tau} Q_{, \theta}^{2}\right)=0, \\
Q_{, \tau \tau}-e^{-2 \tau} Q_{, \theta \theta}+2\left(P_{, \tau} Q_{, \tau}-e^{-2 \tau} P_{, \theta} Q_{, \theta}\right)=0,
\end{gathered}
$$

and two first order differential equations for $\lambda$,

$$
\begin{gathered}
\lambda_{, \tau}=P_{, \tau}^{2}+e^{-2 \tau}+e^{2 P}\left(Q_{, \tau}^{2}+e^{-2 \tau} Q_{, \theta}^{2}\right), \\
\lambda_{, \theta}=2\left(P_{, \theta} P_{, \tau}+e^{2 \tau} Q_{, \theta} Q_{, \tau}\right) .
\end{gathered}
$$

The set of equations for $\lambda$ is the equivalent of Eqs. (33) and can be solved by quadratures once $P$ and $Q$ are known.

As we mentioned, the special case of the polarized $T^{3}$ model is obtained from the metric (134) just by taking $Q=0$. The resulting field equations are easier to handle, and a general solution for the main function $P$ can be found by separation of variables. In fact, let us consider $P(\tau, \theta)=T(\tau) \Theta(\theta)$; then, Eq. (35) separates into

$$
\frac{1}{\Theta} \frac{d^{2} \Theta}{d \theta^{2}}=-n^{2}, \quad \text { and } \quad \frac{d^{2} T}{d \tau^{2}}+n^{2} e^{-2 \tau} T=0
$$

where $n$ is the separation constant which in this case has to be an integer in order for the condition $\Theta(\theta+2 \pi)=\Theta(\theta)$ to be satisfied. With this assumption, the general solution for $P$ can be written as an infinite series of the form

$$
P=\sum_{n=0}^{\infty}\left[A_{n} \cos (n \theta)+B_{n} \sin (n \theta)\right]\left[C_{n} J_{0}\left(n e^{-\tau}\right)+D_{n} N_{0}\left(n e^{-\tau}\right)\right],
$$

where $A_{n}, B_{n}, C_{n}$ and $D_{n}$ are arbitrary constants. If we want to avoid singularities at $\tau=+\infty$, the constant $D_{n}$ has to vanish.

The models described by the general $(Q \neq 0)$ metric (34) have been used extensively for numerical investigations in classical as well as in minisuperspace quantum gravity [59]. One of the reasons why these investigations have used numerical methods is because it is usually 
believed that the set of main field equations (35) and (36) is such a complicated system that analytic solutions would be difficult to find. We will show here that it is possible to generate unpolarized solutions $(Q \neq 0)$ from a given polarized solution $(Q=0)$ by using modern solution generating techniques. Of course, the new solutions will be particular solutions, and the numerical investigations in Refs. [59] were carried out with the aim of studying the general behavior of the models near a singularity, information that no particular solution can give. However, families of particular solutions can give us clues about how to set up numerical solutions.

We can apply the solution generating techniques by writing the field equations in such a way that the symmetries involved can be derived and understood easily. To this end, we first introduce a new "time" coordinate $t=e^{-\tau}$ and a new function $R=R(t, \theta)$ by means of the equations

$$
R_{, t}=t e^{2 P} Q_{, \theta}, \quad R_{, \theta}=t e^{2 P} Q_{, t} .
$$

Then, the field equations (36) and (35) can be expressed as

$$
\begin{gathered}
t^{2}\left(P_{, t t}+\frac{1}{t} P_{, t}-P_{, \theta \theta}\right)+e^{-2 P}\left(R_{, t}^{2}-R_{, \theta}^{2}\right)=0 \\
t e^{P}\left(R_{, t t}+\frac{1}{t} R_{, t}-R_{, \theta \theta}\right)-2\left[\left(t e^{P}\right)_{, t} R_{, t}-\left(t e^{P}\right)_{, \theta} R_{, \theta}\right]=0 .
\end{gathered}
$$

Furthermore, this last equation for $R$ turns out to be identically satisfied if the integrability condition $R_{, t \theta}=R_{, \theta t}$ is fulfilled.

We can now introduce the complex Ernst potential $\epsilon$ and the complex gradient operator $D$ as

$$
\epsilon=t e^{P}+i R, \quad \text { and } \quad D=\left(\frac{\partial}{\partial t}, i \frac{\partial}{\partial \theta}\right),
$$

which allow us to write the main field equations in the Ernst-like representation

$$
\operatorname{Re}(\epsilon)\left(D^{2} \epsilon+\frac{1}{t} D t D \epsilon\right)-(D \epsilon)^{2}=0
$$

It is easy to see that the field equations (42, 43) can be obtained as the real and imaginary part of the Ernst equation (45), respectively.

The importance of this representation is that it also can be derived from a Lagrangian by means of a variational principle. In turn, that Lagrangian may be interpreted as a metric Lagrangian defined in a two dimensional Riemannian space (the potential space), 
the coordinates of which are the real and imaginary parts of the Ernst potential. Applying the variational principle in the potential space on the metric Lagrangian, one obtains the corresponding geodesic equations, which turn out to be equivalent to the Ernst equation (45). Hence, to investigate the symmetries of the Ernst equation one can study infinitesimal transformations which leave invariant the geodesic equations in the the potential space. In particular, the transformations associated with the Killing vector fields of the metric in the potential space leave the corresponding geodesic equations invariant. One could think of a solution of the Ernst equation as a geodesic in the potential space, and the Killing vectors of the metric as transformations that starting from a given geodesic lead to a different geodesic, i.e., to a different solution of the Ernst equation. This is the basic idea behind some of the known solution generating techniques.

We will now derive a simple but illustrative symmetry of the Ernst equations which allows to generate new solutions. To this end, we introduce a new complex potential $\xi=\xi(t, \theta)$ by means of the relationship

$$
\epsilon=\frac{1-\xi}{1+\xi}
$$

Then, the Ernst equation (45) transforms into

$$
\left(1-\xi \xi^{*}\right)\left(D^{2} \xi+\frac{1}{t} D t D \xi\right)+2 \xi^{*}(D \xi)^{2}=0
$$

where $\xi^{*}$ represents the complex conjugate potential. Furthermore, we introduce new coordinates $x$ and $y$ by

$$
t^{2}=c^{2}\left(1-x^{2}\right)\left(1-y^{2}\right), \quad \theta=c x y
$$

where $c$ is a real constant. In these coordinates, the main field equation (47) can be written in the following form

$$
\left(1-\xi \xi^{*}\right)\left\{\left[\left(1-x^{2}\right) \xi_{, x}\right]_{, x}-\left[\left(1-y^{2}\right) \xi_{, y}\right]_{, y}\right\}+2 \xi^{*}\left[\left(1-x^{2}\right) \xi_{, x}^{2}-\left(1-y^{2}\right) \xi_{, y}^{2}\right]=0
$$

which explicity shows the invariance with respect to the change of coordinates $x \leftrightarrow y$, i.e., if $\xi(x, y)$ is a solution of (49) then $\xi(y, x)$ is also a solution. The simplest solution of Eq. (49) is $\xi^{-1}=x$, so $\xi^{-1}=y$ is also a solution. A linear combination of these two solutions

$$
\xi^{-1}=a x+i b y
$$

turns out to also be a solution if the condition $a^{2}+b^{2}=1$ is satisfied. In this way it is relatively easy to generate new solutions. Once the potential $\xi$ is known one can calculate 
the functions $P$ and $R$ algebraically by means of Equations (46) and (44). Then, Eq. (41) may be integrated to yield $Q$. Finally, the solution for the function $\lambda$ may be calculated by quadratures.

It is interesting to note that one can derive some of the main properties of the Gowdy model just by inspecting the explicit form of the Ernst potential. If the Ernst potential $\epsilon$ is real, then the function $R$ vanishes and, therefore, the metric function $Q$ is a constant which can be absorbed by means of a suitable coordinate transformation. Thus, a real Ernst potential corresponds to a polarized Gowdy model. In the case of complex Ernst potential with nontrivial imaginary part (not proportional to its real part), it is guaranteed that the resulting metric corresponds to an unpolarized Gowdy model $(Q \neq 0)$. For instance, even the simple solution (50) will lead to a nontrivial unpolarized solution with $Q \neq 0$.

\section{B. Generation of New Solutions}

In this section we will briefly describe the way new solutions can be generated by using HKX transformations. Let us assume that a solution for the polarized Gowdy model is given by $P=P_{0}(t, \theta)$ and $\lambda=\lambda_{0}(t, \theta)$. The corresponding Ernst potential is real, and we denote it by $\epsilon_{0}=t e^{P_{0}}$.

A HKX transformation acting on $\epsilon_{0}$ generates a new complex Ernst potential $\epsilon$ which will correspond to an unpolarized Gowdy model only if its imaginary part is not proportional to its real part. This will depend on the explicit form of the initial Ernst potential $\epsilon_{0}$. However, if we apply two different HKX transformations it can be shown that the resulting Ernst potential is nontrivial. For this reason, we present here the result of the action of two HKX transformations on the real Ernst potential $\epsilon_{0}$. For the sake of simplicity, we use the coordinates $x$ and $y$ defined in Eq. (48) in which the new Ernst potential can be written as $42]$

$$
\epsilon=\epsilon_{0} \frac{x\left(1-\mu_{1} \mu_{2}\right)+i y\left(\mu_{1}+\mu_{2}\right)+\left(1+\mu_{1} \mu_{2}\right)+i\left(\mu_{1}-\mu_{2}\right)}{x\left(1-\mu_{1} \mu_{2}\right)+i y\left(\mu_{1}+\mu_{2}\right)+\left(1+\mu_{1} \mu_{2}\right)-i\left(\mu_{1}-\mu_{2}\right)},
$$

where we have introduced new functions $\mu_{1}$ and $\mu_{2}$ defined by

$$
\mu_{1}=\alpha_{1} e^{2 \beta_{-}} \quad \text { and } \quad \mu_{2}=\alpha_{2} e^{2 \beta_{+}}
$$

Here $\alpha_{1}$ and $\alpha_{2}$ are real constants and $\beta_{ \pm}$are functions which satisfy a set of two first order 
differential equations

$$
\begin{aligned}
& (x \mp y)\left(\beta_{ \pm}\right)_{, x}=(1 \mp x y) \frac{\epsilon_{0, x}}{\epsilon_{0}} \mp\left(1-y^{2}\right) \frac{\epsilon_{0, y}}{\epsilon_{0}}, \\
& (x \mp y)\left(\beta_{ \pm}\right)_{, y}=(1 \mp x y) \frac{\epsilon_{0, x}}{\epsilon_{0}} \pm\left(1-x^{2}\right) \frac{\epsilon_{0, y}}{\epsilon_{0}} .
\end{aligned}
$$

Thus, the generation of new solutions reduces to the integration of the differential equations for $\beta_{ \pm}$. The new constants $\alpha_{1}$ and $\alpha_{2}$ have been introduced by the two HKX transformations. For vanishing $\alpha_{1}$ and $\alpha_{2}$, the potential (51) reduces to the original Ernst potential of the polarized Gowdy model.

Equations (53) and (54) allow us to generate new unpolarized solutions starting from a given polarized Gowdy model. The derivation of explicit solutions implies the integration of a set of two first order differential equations for a function which determines the Ernst potential of the new solution. In turn, from the Ernst potential one can obtain the new metric functions which completely determine the spacetime of the new cosmological model. In order to complete this procedure it is necessary to carry out lenghtly but straightforward calculations. We are attempting to calculate the unpolarized solution corresponding to the general solution (40) of the polarized $T^{3}$ Gowdy model.

\section{CONCLUSIONS AND SUGGESTIONS FOR FURTHER RESEARCH}

We have given several examples of the three methods listed in the Introduction. It is obvious that these three approaches are far from exhausted, and may give a number of interesting exact solutions to known types of cosmological models and suggest new types of models of interest. It is probable that the horizon and mapping methods may be more productive than causal structure methods.

It seems that horizon methods have barely touched the surface of possible solutions. There are a large number of exact solutions of "black hole" type given in Ref. [46] that are candidates for generating cosmological models inside their horizons. We have looked at the Kantowski-Sachs-Schwarzschild, Taub-NUT and Kerr manifolds in some detail. We have also mentioned as possibilities the Tomimatsu-Sato and Quevedo class of solutions which should lead to models with mixed singularities, part null surface and part curvature singularity. There are large classes of vacuum type D solutions that might give interesting 
cosmologies [46]. Even such well known solutions as Kerr-Taub-NUT do not seem to have been investigated thoroughly as cosmological models.

Models with matter seem hardly to have been touched. While fluid models may be difficult to generate, the example of electrovac universes shows us that there are many possible cosmologies of this type. Even the interior of the Reissner-Nordstrøm solution does not seem to have been investigated in this context. It would also be interesting to investigate the possibility of obtaining useful cosmological models from the most general type D electrovac solutions with cosmological constant, found by Debever et al. [61], which contains 13 different parameters and includes the Kerr-Newman solution as a special case. Even the Kerr-Newman solution [13] would generate an $R^{1} \times S^{2}$ (and even, perhaps, an $S^{1} \times S^{2}$ ) Gowdy solution, equivalent to, or a generalization of, the solutions of Carmeli, Charach and Malin [60] for $T^{3}$ models. Other types of solutions, such as scalar field models do not seem to have been investigated at all in this context.

If anything, mapping methods seem even more underutilized in cosmology. The example we have seen is one of many that come to mind. For the Gowdy models one could use these methods to investigate the $S^{1} \times S^{2}$ models and perhaps generate unkown solutions. The corresponding Ernst potential of the field equations present some technical difficulties related to the specific topology of the model. This problem is currently under investigation. For this case, it would also be interesting to generate new unpolarized solutions starting from the general polarized solution, which in the case of $S^{1} \times S^{2}$ models, just as in the $T^{3}$ models, can also be represented as an infinite series of eigenfunctions since the corresponding field equations reduce to separable linear second order differential equations.

Solutions with with true curvature singularities on possible inner and outer horizons might be especially interesting. An example of this idea was given by Moncrief [62], who used the Geroch group to generate a solution that had a curvature singularity in place of a horizon in the Kerr-Taub-NUT case.

There will certainly be many opportunities to apply all three of the techniques discussed in the article to the generation of new cosmologies. 


\section{Acknowledgments}

We wish to thank T. Jacobson, V. Moncrief and O. Obregon for useful discussions. This work was supported in part by CONACyT grant 3567E, and DGAPA-UNAM grants 121298 and IN106097.

[1] Krasiński A. (1997) Inhomogeneous Cosmological Models. Cambridge U. P., Cambridge.

[2] The literature on the deSitter metric is vast. For the description given here see: Hawking S., Ellis G. (1973) The Large Scale Structure of Space-Time. Cambridge U. P., Cambridge; Møller C. (1952) The General Theory of Relativity. Oxford U. P., Oxford.

[3] deSitter W. (1917) Mon. Not. Roy. Astron. Soc. 78, 3.

[4] Lemaitre G. (1925) J. Math. and Phys. (M.I.T.) 4, 188.

[5] Robertson H. (1928) Phil. Mag. 5, 835.

[6] Gowdy R. (1971) Phys. Rev. Lett. 27, 826; (1974) Ann. Phys. (N.Y.) 83, 203.

[7] Bondi H., Pirani F., Robinson I. (1959) Proc. Roy. Soc. London A251, 519; Ehlers J., Kundt W., (1962) in: Witten L. (Ed.) Gravitation: An Introduction to Current Research. Wiley, New York.

[8] Kerr R. P. (1963) Phys. Rev. Lett. 11, 237.

[9] Newman E. T., Tamburino L. and Unti T. (1963) J. Math. Phys. 4, 915.

[10] Kinnersley W. (1969) J. Math. Phys. 10, 1195.

[11] Plebański J. F. and Demiański M. (1976) Ann. Phys.(N. Y.) 98, 98.

[12] Newman E. T. and Janis A. I. (1965) J. Math. Phys. 6, 915.

[13] Newman E. T. et al. (1965) J. Math. Phys. 6, 918.

[14] Demiański M. and Newman E. T. (1966) Bull. Acad. Pol. Sci., Ser. Math. Astron. Phys. 14, 653.

[15] Carter B. (1968) Comm. Math. Phys. 10, 280.

[16] Demiański M. (1973) Acta Astronom. Pol. 23, 197.

[17] Ernst F. J. (1968) Phys. Rev. 167, 1175.

[18] Ernst F. J. (1968) Phys. Rev. 168, 1415.

[19] Kinnersley W. (1973) J. Math.. Phys. 14, 651. 
[20] Kinnersley W. (1977) J. Math. Phys. 18, 1529.

[21] Tomimatsu A. and Sato H. (1972) Phys. Rev. Lett. 29, 1344.

[22] Tomimatsu A. and Sato H. (1973) Prog. Theor. Phys. 50, 95.

[23] Yamazaki M. and Hori S. (1977) Prog. Theor. Phys. (Lett.) 57, L696.

[24] Yamazaki M. (1977) Prog. Theor. Phys. 57, 1951.

[25] Yamazaki M. (1977) J. Math. Phys. 18, 2502.

[26] Geroch R. (1971) J. Math. Phys. 12, 918.

[27] Geroch R. (1972) J. Math. Phys. 13, 394.

[28] Cosgrove C. M. (1977) J. Phys. A: Math. Gen. 10, 1481.

[29] Cosgrove C. M. (1977) J. Phys. A: Math. Gen. 10, 2093.

[30] Kinnersley W. and Chitre D. M. (1977) J. Math. Phys. 18, 1538.

[31] Kinnersley W. and Chitre D. M. (1978) J. Math. Phys. 19, 1926.

[32] Kinnersley W. and Chitre D. M. (1978) J. Math. Phys. 19, 2037.

[33] Kinnersley W. and Chitre D. M. (1977) Phys. Rev. Lett. 40, 1608.

[34] Hoenselaers C., Kinnersley W., and Xanthopoulos B. C. (1979) Phys. Rev. Lett. 42, 481.

[35] Hoenselaers C., Kinnersley W., and Xanthopoulos B. C. (1979) J. Math. Phys. 20, 2530.

[36] Harrison B. K. (1978) Phys. Rev. Lett. 41, 1197.

[37] Neugebauer G. (1980) J. Phys. A: Math. Gen. 13, 1737, L19.

[38] Belinsky V. A. and Zakharov V. E. (1978) Soviet Phys.-JETP 48, 985.

[39] Belinsky V. A. and Zakharov V. E. (1978) Soviet Phys.-JETP 50, 1.

[40] Cosgrove C. M. (1980) J. Math. Phys. 21, 2417.

[41] Cosgrove C. M. (1982) in: Proceedings of the Second Marcel Grossmann Meeting on General Relativity, Ruffini R. (Ed.), North-Holland, Amsterdam.

[42] Quevedo H. (1990) Fortschr. Phys. 38, 10.

[43] See, for example, Rindler W. (1969) Essential Relativity: Special, General, and Cosmological (Second Ed.). Springer-Verlag, Heidelberg.

[44] Taub A. (1951) Ann. Math. 53, 472.

[45] Misner C. (1963) in: Relativity Theory and Astrophysics 1. Ehlers J. (Ed.), American Mathematical Society, Providence R. I.; Misner C., Taub A. (1968) Zh. Eksp. Teor. Fiz. 55, 233 (Soviet Physics - JETP 28, 122 [1969]).

[46] Kramer D., Stephani D., Herlt E., MacCallum M., Schmutzer E. (1980) Exact Solutions of 
Einstein's Field Equations. Cambridge U. P., Cambridge.

[47] See Burko L., Ori A. (Eds.) (1997) Proceedings of the Workshop on the Internal Structure of Black Holes and Spacetime Singularities. IOP, Bristol.

[48] Obregón O., Ryan M. (1998) gr-qc/9810068

[49] Ryan M., Shepley L. (1975) Homogeneous Relativistic Cosmologies. Princeton U. P., Princeton.

[50] Conradi H-D. (1995) Class. Q. Grav. 12, 2423.

[51] Brill D. (1964) Phys. Rev. 133, B845.

[52] Israel W. (1967) Phys. Rev. 164, 1776; Carter B. (1970) Phys. Rev. Lett. 26, 331; Hawking S. (1972) Commun. Math. Phys. 25, 152.

[53] Quevedo H. (1986) Phys. Rev. D 33, 324.

[54] Einstein A., Rosen N. (1937) J. Franklin Inst. 223, 43.

[55] Jacobson T. (1998) Private communication.

[56] Laflamme R., Shellard E. P. S. (1987) Phys. Rev. D 35, 2315.

[57] Xanthopolous B., Zannias T. (1992) J. Math. Phys. 33, 1415; (1991) J. Math. Phys. 30, 1121.

[58] Hawking S., Ellis G. in Ref. 2].

[59] See Berger B., Moncrief V. (1993) Phys. Rev. D 48, 4676; Berger B., Garfinkle D. (1998) Phys. Rev. D 57, 4767, and references therein.

[60] Charach Ch. (1979) Phys. Rev. D 19, 1058; Phys. Rev. D 19, 3516; Carmeli M., Charach Ch., Malin S. (1981) Phys. Reports 76, 1.

[61] Debever R., Kamran N., McLenaghan R. G. (1984) J. Math. Phys. 25, 1955.

[62] Moncrief V. (1987) Class. Q. Grav. 6, 1555. 